\title{
Effect of recombinant adeno-associated virus expressing calcitonin gene-related peptide on chick embryo umbilical artery vasospasm model
}

\author{
YONGJIE YUAN, SI YANG, CHAO LI, KAN XU and JINLU YU \\ Department of Neurosurgery, First Hospital of Jilin University, Changchun, Jilin 130021, P.R. China
}

Received November 25, 2015; Accepted November 25, 2016

DOI: $10.3892 /$ etm.2017.5423

\begin{abstract}
In the present study, a recombinant adeno-associated virus vector containing the calcitonin gene related peptide gene (rAAV-CGRP) was constructed and the therapeutic effect of rAAV-CGRP on a chick umbilical artery vasospasm model induced by chick embryo allantoic cavity hemorrhage was investigated. Fresh specific pathogen-free fertilized chicken eggs were randomly divided into a rAAV-CGRP group, an empty vector virus (AAV) group, and a control group, with 24 eggs in each group. An umbilical arterial vasospasm model was established using a needle puncture method on a vein in the chorioallantoic membrane to induce a hemorrhage in the allantoic cavity of 11-day-old chicken embryonated eggs. A total of $24 \mathrm{~h}$ after model establishment, $1 \mathrm{ml}$ of rAAV-CGRP and empty vector virus solution of rAAV-CGRP and empty vector virus solution was, respectively, injected into the allantoic cavity in the rAAV-CGRP and AAV groups. Experimental results showed that after $72 \mathrm{~h}$ of model establishment, the mortality rates of the 3-, 5- and 7-day subgroups in the rAAV-CGRP group were lower than in the subgroups of the AAV injection group. After 3,5 and 7 days of model establishment in the rAAV-CGRP group, the cross-sectional area of the inner diameter of the umbilical arteries was larger than that of the AAV group; the vessel wall thicknesses of the rAAV-CGRP group were thinner than in the AAV group. In addition, the concentration of CGRP in chick embryo allantoic fluid significantly increased and was several times higher than in the AAV group $(\mathrm{P}<0.05)$. In conclusion, administration of rAAV-CGRP through the allantoic cavity may increase the viability of a vasospasm model induced by chick allantoic cavity hemorrhage, significantly improve umbilical artery vasospasm, and increase CGRP expression in the chick embryo allantoic cavity. This approach also provides a novel
\end{abstract}

Correspondence to: Professor Jinlu Yu, Department of Neurosurgery, First Hospital of Jilin University, 71 Xinmin Avenue, Changchun, Jilin 130021, P.R. China

E-mail: jinluyu@hotmail.com

Key words: adeno-associated virus, calcitonin gene related peptide, gene therapy, vasospasm experimental model for identifying other target genes for the gene therapy of vasospasm.

\section{Introduction}

Subarachnoid hemorrhage (SAH) is a common disease in neurosurgery, accounting for $10 \%$ of acute strokes and $20 \%$ of hemorrhagic strokes around the world $(1,2)$. Ischemic brain damage secondary to cerebral vasospasm (CVS) is a common and dangerous complication of SAH and is one of the most common reasons for morbidity and mortality after SAH (1-3). The prevention and treatment of cerebral vasospasm primarily relies on nimodipine administration and optimization of blood volume and cardiac performance, pharmacologically induced hypertension combined with volume is the established first-line therapy for delayed cerebral vasospasm (4). However, occasionally medically refractory delayed cerebral vasospasm may occur, defined as failure to respond adequately to these measures (5). Although there are multiple domestic and international studies focusing on the mechanism underlying the development of CVS after SAH, its pathophysiological basis has not been fully elucidated. Studies have demonstrated that a large amount of calcitonin gene-related peptide (CGRP) is released from nerve endings into human blood to oppose vasospasm at the early stage of CVS (6-8). With the progression of time, CGRP at nerve endings is depleted, and CVS develops progressively. It is speculated that the depletion of endogenous CGRP may be a key reason for CVS development after SAH. As a neuropeptide containing 37 amino acids, CGRP has strong vasodilation and neuronal protection functions $(6,9)$. Animal experiments and early clinical trials have shown that intra-cisternal injection or intravenous injection of exogenous CGRP is able to significantly relieve vasospasm and improve nerve function (10-14). However, due to its protein features, CGRP has a very short plasma half-life of only 7-10 min, which restricts its clinical application (15).

In the present study, genetic engineering technology was used to clone the CPGR gene into the adeno-associated virus (AAV) to construct a eukaryotic expressing vector containing the CGRP gene (rAAV-CGRP). Through the allantoic cavity injection approach, the feasibility of using rAAV-CGRP to improve umbilical artery vasospasm in the chick embryo allantoic cavity was demonstrated. 


\section{Materials and methods}

Experimental grouping. A total of 80 specific pathogen-free fresh fertilized chicken eggs (Merial Vital Laboratory Animal Technology, Beijing, China) were selected. The mean weight was $60 \pm 2.9 \mathrm{~g}$. Eggs were randomly divided into the rAAV group, the empty vector AAV group, and the control group, with 24 eggs in each group. Other eggs were used as a reserve supply. Each group was further randomly divided into three subgroups: 3-, 5- and 7-day groups, with 8 eggs in each subgroup. Fertilized chicken eggs were hatched in an automatic incubator. Hatching parameters were as follows: Temperature, $37.8^{\circ} \mathrm{C}$; humidity, $60 \%$; and rotation of eggs, once every $90 \mathrm{~min}$. After five days of hatching, the chick embryos were observed under an egg-candling lamp in a dark room. A total of three chick embryos failed to hatch were excluded and replaced with fertilized eggs from the reserve supply. All animal experimental operations were approved by the Ethics Committee of Jilin University (Changchun, China).

Establishment of an umbilical artery vasospasm model in the chick embryo allantoic cavity. A previous study by our group confirmed that allantoic cavity hemorrhage caused by puncture of chorioallantoic membrane (CAM) blood vessels can induce umbilical artery vasospasm that presents certain features of the pathological changes of CVS after SAH in mammals (16). For model establishment, fertilized chicken eggs at 11 days of age were selected. Chick embryos were observed under an egg-candling lamp in a dark room through the blunt end of the air chamber. CAM blood vessels in chick embryos were clearly observed. At the top of the chicken embryo, a large vein departed the CAM, after multiple vascular branches congregated, and extended toward the deep allantoic cavity to connect to the chicken embryo, which is the umbilical vein. A relatively large CAM vessel was selected for needle puncturing. The surface of the fertilized eggs was wiped with alcohol-moistened cotton balls. The eggshell was initially drilled using a 16-G syringe needle; the CAM and blood vessels were not damaged. The CAM blood vessel was subsequently punctured using a $26-\mathrm{G}$ syringe needle. Obvious bleeding was observed under an egg-candling lamp. The fertilized egg was gently rotated to evenly disperse the blood in the allantoic cavity, which made the allantoic fluid turbid. In the control group, the eggshell was only drilled using a 16-G syringe needle, and the CAM vein was not punctured. The eggs were returned to the incubator for hatching until being sacrificed (see Sample collection and fixation of umbilical arteries in the allantic cavity) at 3,5 and 7 days, respectively. Each egg was exposed to an egg-candling lamp every day to remove chick embryos that did not survive.

Construction and vaccination of $r A A V$. The construction of the chick rAAV-CGRP virus is described in previous literature. Briefly, the forward and reverse primers of chick CGRP were designed and synthesized by Sangon Biotech, Co., Ltd. (Shanghai, China; forward, 5'-CCGGAATTCATGGTCATG CTGAAGATTTCATC-3' and reverse, 5'-CAAGCTTCTAGT TGTTTCCTAGGGTTTCCCCA-3'). Using polymerase chain reaction with Takara Ex Taq DNA polymerase (Takara Bio, Inc., Otsu, Japan) with a 7500 Fast thermocycler (Applied
Biosystems; Thermo Fisher Scientific, Inc., Waltham, MA, USA), the fragment encoding CGRP was produced, including the EcoRI and HindIII restriction enzyme sites. The PCR procedures as follow: Denaturation at $94^{\circ} \mathrm{C}$ for $60 \mathrm{sec}$, annealing at $37^{\circ} \mathrm{C}$ for $60 \mathrm{sec}$, extension at $72^{\circ} \mathrm{C}$ for $80 \mathrm{sec}$ for 30 cycles, followed by $5 \mathrm{~min}$ at $72^{\circ} \mathrm{C}$ for the final extension. Next, the synthesized fragments were added to the pGEM-T-Easy vectors (Promega Corporation, Madison, WI, USA), and the connection products were transformed into Escherichia coli TOP10 bacterial strains. The positive clone was identified using EcoRI and HindIII restriction enzymes (Takara Bio, Inc., Otsu, Japan), and the cloned amplified fragments were sequenced by the dideoxy-mediated chain-termination method. Cloned CGRP cDNAs were compared with the GenBank sequence using DNASIS software (Version 2.7; MiraiBio Group, San Francisco, CA, USA). Subsequently, pGEM-T-CGRP and shuttle vector PSSCMV (Huaguang, China) were digested by EcoRI and HindIII, respectively. Once treated with T4 DNA ligase, CGRP and linearized pSS-CMV were recovered on a low melting point gel, and the novel products were transformed into E. coli DH5a-competent cells. Eventually, the recombinant vectors were collected and digested by EcoRI and HindIII, and the positive clones were subsequently identified by advanced glycation end product. To acquire the rAAV, HEK293 cells were co-transfected with pSSCMV-CGRP and aid vector (Helper plasmid, to provide protein necessary for AVV replication and packaging) pAAV-Ad by calciumphosphate co-precipitation. After three days, the rAAV was collected from the supernatant by repeatedly freezing/thawing the culture medium containing the virus embolus. HEK293 cells were treated by multiple dilutions of $\mathrm{rAAV}$, and the virus titer was estimated by dot-blot hybridization (17). The virus titer of the recombinant group was $1.15 \times 10^{12} \mathrm{pfu} / \mathrm{ml}$. The titer of the empty vector virus in the empty vector AAV group was $1.13 \times 10^{12} \mathrm{pfu} / \mathrm{ml}$. Syringes were used to inject $1 \mathrm{ml}$ of recombinant virus solution or empty vector virus solution into the chick embryo allantoic cavity after $24 \mathrm{~h}$ of model establishment. The pSSCMV viral vector, adenovirus vector $\mathrm{pAAV} / \mathrm{Ad}$, E. coli TOP10, E. coli DH5 $\alpha$ and HEK293 cell lines were provided by Xi'an Huaguang Biological Engineering Co., Ltd., (Xi'an, China).

Detection of CGRP concentrations in the allantoic fluid using ELISA. On days 3, 5 and 7 after model establishment, fertilized eggs were broken from the air chamber end with tweezers to expose the inner shell membrane and the tightly attached CAM below. Cotton swabs dipped in PBS were used to gently wipe the inner shell membrane. The CAM blood vessels inside could be clearly seen. Caution was taken to avoid the large vessels as the inner eggshell membrane and the attached CAM was gently torn up with a $16-\mathrm{G}$ syringe needle to enter the allantoic cavity. Allantoic fluid was aspirated using a syringe, and the CGRP levels in the allantoic fluid were detected using a chicken CGRP ELISA kit (MBS2513672; MyBioSource, San Diego, CA, USA). Procedures were performed according to the instruction manual provided with the reagent kit. Standard concentrations were assigned to the horizontal axis, and optical density (OD) values were assigned to the vertical axis. The standard curve was plotted on coordinate paper, and the linear 
regression equation of the standard curve was calculated. The OD values of the samples were introduced into the equation to calculate the sample concentrations. The results multiplied by the dilution fold were the actual sample concentrations.

Sample collection and fixation of umbilical arteries in the allantoic cavity. On days 3, 5 and 7 after model establishment, the fertilized eggs were carefully opened from the air chamber end using forceps to expose the shell membrane and the tightly attached CAM below. Cotton swabs dipped in PBS were used to gently wipe the inner shell membrane. Inside, the CAM blood vessels could be clearly observed. Care was taken to avoid the large vessels as the inner eggshell membrane and the attached CAM was gently torn up with a $16-\mathrm{G}$ syringe needle to enter the allantoic cavity. Allantoic fluid was aspirated as much as possible. A syringe was used to inject $4 \%$ paraformaldehyde (in $0.1 \mathrm{~mol} / 1 \mathrm{PBS}$; $\mathrm{pH} 7.4$ ), precooled at $4^{\circ} \mathrm{C}$, into the allantoic cavity for fixation at $4^{\circ} \mathrm{C}$ for $2 \mathrm{~h}$. The eggshell was opened and the umbilical arteries in the allantoic cavity that were connected to the ventral side of the chick embryo could be observed. Subsequently, $\sim 1 \mathrm{~cm}$ of the proximal end of the right branch of the umbilical artery after bifurcation was collected and placed in $4 \%$ paraformaldehyde for fixation and storage.

Measurement of the cross-sectional area (CSA) of the inner diameter and vessel wall thickness of the umbilical arteries. The paraformaldehyde-fixed umbilical artery was evenly divided into three segments (proximal, distal and middle segments), dehydrated, cleared and embedded. Cross sections of the blood vessels were prepared at a $5-\mu \mathrm{m}$ thickness; sections were stained with hematoxylin and eosin, observed, and images were captured under a microscope at x200 magnification (Olympus Corp., Tokyo, Japan). The CSA of the inner and outer diameters of the blood vessels was measured separately by two individuals using ImageJ software (Version 1.48; National Institutes of Health, Bethesda, MA, USA). The mean value of the CSA of three segments was obtained, and the mean value of the measurement results from the two individuals was subsequently calculated. Vessel wall thickness was calculated using a geometric formula according to the CSA of the inner and outer diameters.

Statistical analysis. SPSS 21.0 statistical software (IBM SPSS, Armonk, NY, USA) was used for statistical analysis. Measurement data are presented as the mean \pm standard deviation. Comparison of measurement data between two groups was performed using the Student's t-test. Countable data were compared using the Chi-square test. $\mathrm{P}<0.05$ was considered to indicate a statistically significant difference.

\section{Results}

Mortality rate of the umbilical artery vasospasm model in the chick embryo allantoic cavity after $72 \mathrm{~h}$. At $72 \mathrm{~h}$ after model establishment, only one chick embryo in the 7-day subgroup of the control group did not survive. Following rAAV-CGRP injection, the mortality rates in the 3-, 5- and 7-day subgroups were $25 \%(2 / 8), 25 \%(2 / 8)$ and $37.5 \%(3 / 8)$, respectively, which were lower than the values of $50 \%(4 / 8), 37.5 \%(3 / 8)$

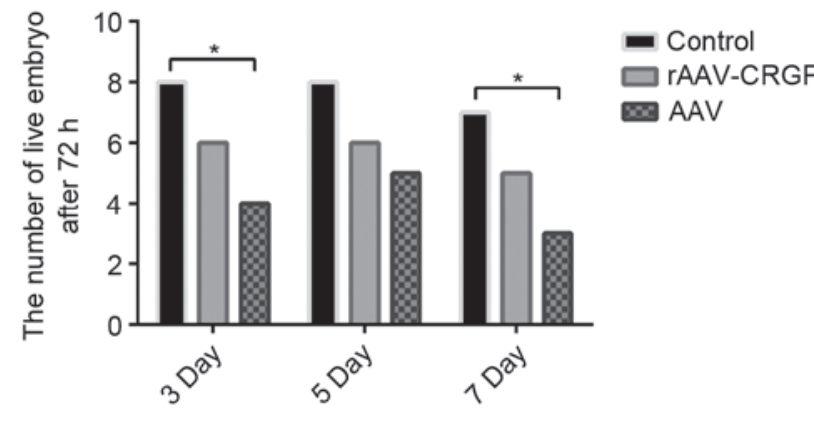

Figure 1. Number of live chick embryos in all subgroups at $72 \mathrm{~h}$ after model establishment. One chick embryo did not survive in the 7-day subgroup of the control group. Mortality rates were 2, 2 and 3 in the 3-, 5- and 7-day subgroups of the rAAV-CGRP group, respectively. Mortality rates in the 3-, 5- and 7-day subgroups of the empty vector AAV group were 4, 5 and 3, respectively. ${ }^{*} \mathrm{P}<0.05$. rAAV, recombinant adeno-associated virus; CGRP, calcitonin gene-related peptide; AAV, adeno-associated virus.

and $62.5 \%(5 / 8)$ in the 3-, 5- and 7-day subgroups after AAV injection. There was no statistical significance between these two groups $(\mathrm{P}>0.05)$. Notably, the mortality rates in the 3- and 7-day subgroups of the chick embryo hemorrhage model after AAV injection were significantly higher than those in the control group $(\mathrm{P}<0.05$; Fig. 1).

Improvement of vasospasm after rAAV-CGRP transfection. Inner CSA and vessel wall thickness of the umbilical artery in each group are presented in Tables I and II respectively. Typical sections of each group are presented in Fig. 2. Compared with the control group, the inner CSA in the AAV group markedly decreased, and the vessel wall became thicker and exhibited wave-form changes. Compared with the AAV group, the inner CSA in the rAAV-CGRP group increased, and the vessel wall thickening displayed a certain degree of improvement; however, compared with the control group, the inner CSA of the rAAV-CGRP group decreased and the vessel wall became thicker. The inner CSA and vessel wall thickness of the umbilical artery in the chick embryo allantoic cavity of each group are illustrated in Fig. 3A and B. These results revealed that the AAV model group exhibited severe vasospasm of the umbilical artery in the chick embryo allantoic cavity. In addition, the inner CSA values of the umbilical artery were only $67.33,59.19$ and $57.22 \%$, respectively, of the CSA of those in the age-matched control group (Fig. 3C), and the umbilical artery vessel wall thickness increased by 12.17 , 17.59 and $18.95 \%$, respectively, of those in the age-matched control group (Fig. 3D). Compared with the AAV group, the degrees of vasospasm in the 3-, 5- and 7-day subgroups of the rAAV-CGRP group after model establishment significantly improved $(\mathrm{P}<0.05)$; the CSA values of the umbilical arteries were $86.11,79.75$ and $77.60 \%$, respectively, of the CSA of those in the age-matched control group (Fig. 3C). The vessel wall thickness values of the umbilical arteries increased by $5.00,8.21$ and $9.73 \%$ in the 3-, 5- and 7-day subgroups of the rAAV-CGRP group, respectively, of those in the age-matched control group (Fig. 3D). However, compared with the control group, rAAV-CGRP did not completely reverse the vasospasm induced by allantoic cavity hemorrhage caused by puncture. These findings indicated that the CSA and vessel wall 
Table I. Inner CSA $\left(\mu \mathrm{m}^{2}\right)$ of the umbilical artery in each group.

\begin{tabular}{lcrr}
\hline Group & 3 day & 5 day & 7 day \\
\hline Control & $80193.62 \pm 3059.49$ & $150329.14 \pm 2989.76$ & $155615.73 \pm 3202.46$ \\
rAAV-CGRP & $69056.64 \pm 2093.75^{\mathrm{a}}$ & $119891.61 \pm 2095.39^{\mathrm{a}}$ & $120762.51 \pm 3001.47^{\mathrm{a}}$ \\
AAV & $53992.81 \pm 2398.62^{\mathrm{b}}$ & $88976.16 \pm 2207.95^{\mathrm{b}}$ & $89045.38 \pm 2309.93^{\mathrm{b}}$
\end{tabular}

${ }^{\mathrm{a}} \mathrm{P}<0.05$ vs. the control group, ${ }^{\mathrm{b}} \mathrm{P}<0.05$ vs. the $\mathrm{AAAV}-\mathrm{CGRP}$ and control groups. $\mathrm{AAV}$, empty vector virus group.

Table II. Vessel wall thickness of the umbilical artery in each group $(\mu \mathrm{m})$.

\begin{tabular}{lccc}
\hline Group & 3 day & 5 day & 7 day \\
\hline Control & $30.99 \pm 1.13$ & $44.07 \pm 1.83$ & $45.44 \pm 1.82$ \\
rAAV-CGRP & $32.54 \pm 1.37^{\mathrm{a}}$ & $47.69 \pm 1.75^{\mathrm{a}}$ & $49.86 \pm 1.91^{\mathrm{a}}$ \\
AAV & $34.76 \pm 1.61^{\mathrm{b}}$ & $51.82 \pm 1.80^{\mathrm{b}}$ & $54.05 \pm 1.79^{\mathrm{b}}$ \\
\hline
\end{tabular}

${ }^{\mathrm{a}} \mathrm{P}<0.05$ vs. the control group, ${ }^{\mathrm{b}} \mathrm{P}<0.05$ vs. the rAAV-CGRP and control groups. AAV, empty vector virus group.

thickness values of the age-matched umbilical arteries among these three groups displayed significant differences.

Increase in CGRP release in the allantoic fluid after rAAV-CGRP transfection. The chick embryos were opened, and the allantoic fluid was collected from the chick embryos of all groups. CGRP concentrations in the chick embryo allantoic fluid on days 3,5 and 7 after model establishment were detected using ELISA reagent kits. The AAV group exhibited very low levels of CGRP. The CGRP concentration in the rAAV-CGRP group significantly increased $(\mathrm{P}<0.05)$ to be six-fold higher than that of the AAV group (Fig. 4).

\section{Discussion}

CGRP, which is a polypeptide containing 37 amino acids, is a translated product of calcitonin gene expression in nerve tissues. Mature CGRP is currently the strongest known endogenously active vasodilator substance $(6,7,9)$. Studies have revealed that, CGRP is released into the blood from nerve endings at the early stage of CVS after SAH to oppose vasospasm and thereby maintain the blood supply in the brain; therefore, CGRP concentrations in the blood and cerebrospinal fluid (CSF) are increased (18-20). With disease progression, CGRP levels in nerve endings are depleted and CGRP concentrations in the blood gradually decrease, leading to the progressive aggravation of CVS. It has been confirmed that CGRP fibers significantly decrease in a CVS animal model $(21,22)$. These studies support the hypothesis that the synthesis and release of CGRP is closely associated with vasospasm after SAH. Depletion of endogenous CGRP may be an important factor for CVS development after SAH. Exogenous administration of CGRP in animal models also confirmed that CGRP is able to improve CVS after SAH. Nozaki et al (10)

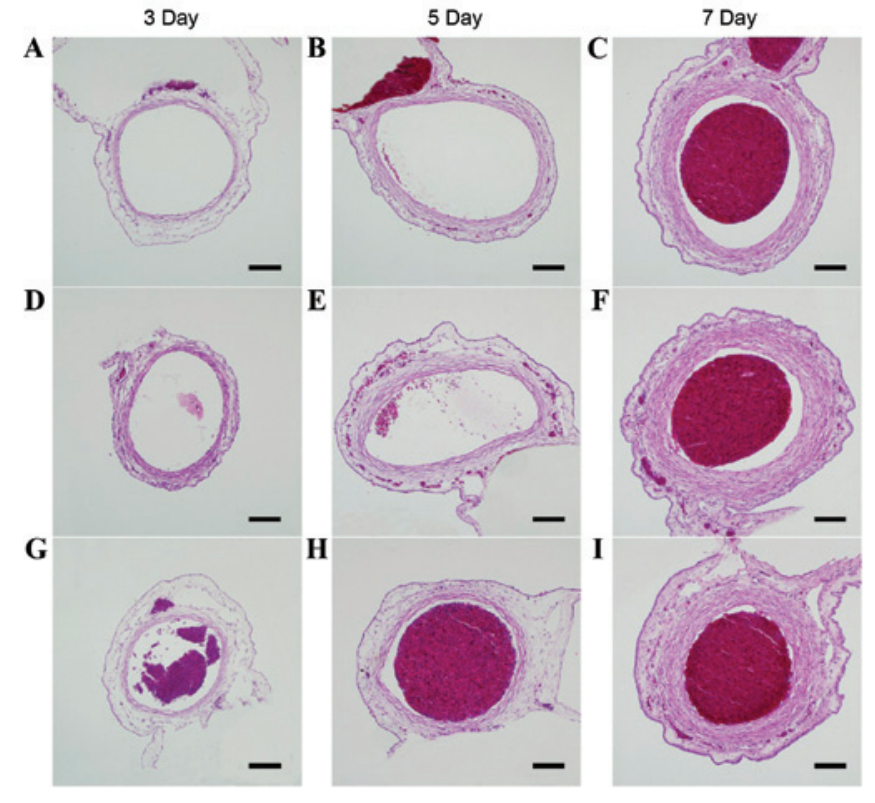

Figure 2. Typical pathological sections of umbilical arteries in all subgroups. Typical pathological sections of umbilical arteries in the allantoic cavity in the (A) 3-, (B) 5- and (C) 7-day subgroups of the control group. Typical pathological sections of umbilical arteries in the allantoic cavity in the (D) 3-, (E) 5- and (F) 7-day subgroups of the rAAV-CGRP group. Typical pathological sections of umbilical arteries in the allantoic cavity in the (G) 3-, (H) 5- and (I) 7-day subgroups of the empty vector AAV group. Compared with structures in the control group, the lumen in the AAV group markedly decreased, and the vessel wall became thicker and displayed a wave-form change. Compared with structures in the AAV group, the lumen in the rAAV-CGRP group became thinner, and the vessel wall thickening exhibited a certain degree of improvement. However, compared with structures in the control group, the lumen still became thinner, and the vessel wall became thicker. Scale bar, $100 \mu \mathrm{m}$. rAAV, recombinant adeno-associated virus; AAV, adeno-associated virus; CGRP, calcitonin gene-related peptide.

conducted an intra-cisternal injection of CGRP on day 3 after the establishment of a cerebral arterial spasm model in dogs and showed that basilar artery spasm improved within $5 \mathrm{~min}$ of injection. Between the doses of $10^{-11}$ and $2 \times 10^{-10} \mathrm{~mol} / \mathrm{kg}$, the effect was even more prominent as the dosage increased; in addition, spasms were completely reversed at $2 \times 10^{-10} \mathrm{~mol} / \mathrm{kg}$. Toshima et al (11) demonstrated that both intra-cisternal and intravenous administration of CGRP improves CVS in rabbits; however, intravenous administration induced significant hypotension. Ahmad et al (23) and Inoue et al (24) implanted a CGRP slow-release tablet in to the cisterna magna to extend the duration of action of CGRP in animal CVS models. In addition, the early clinical trials of intravenously administered exogenous CGRP into CVS patients after SAH confirmed 


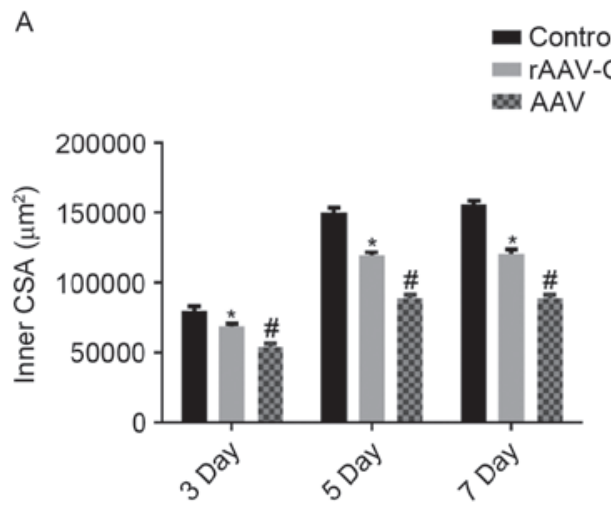

B
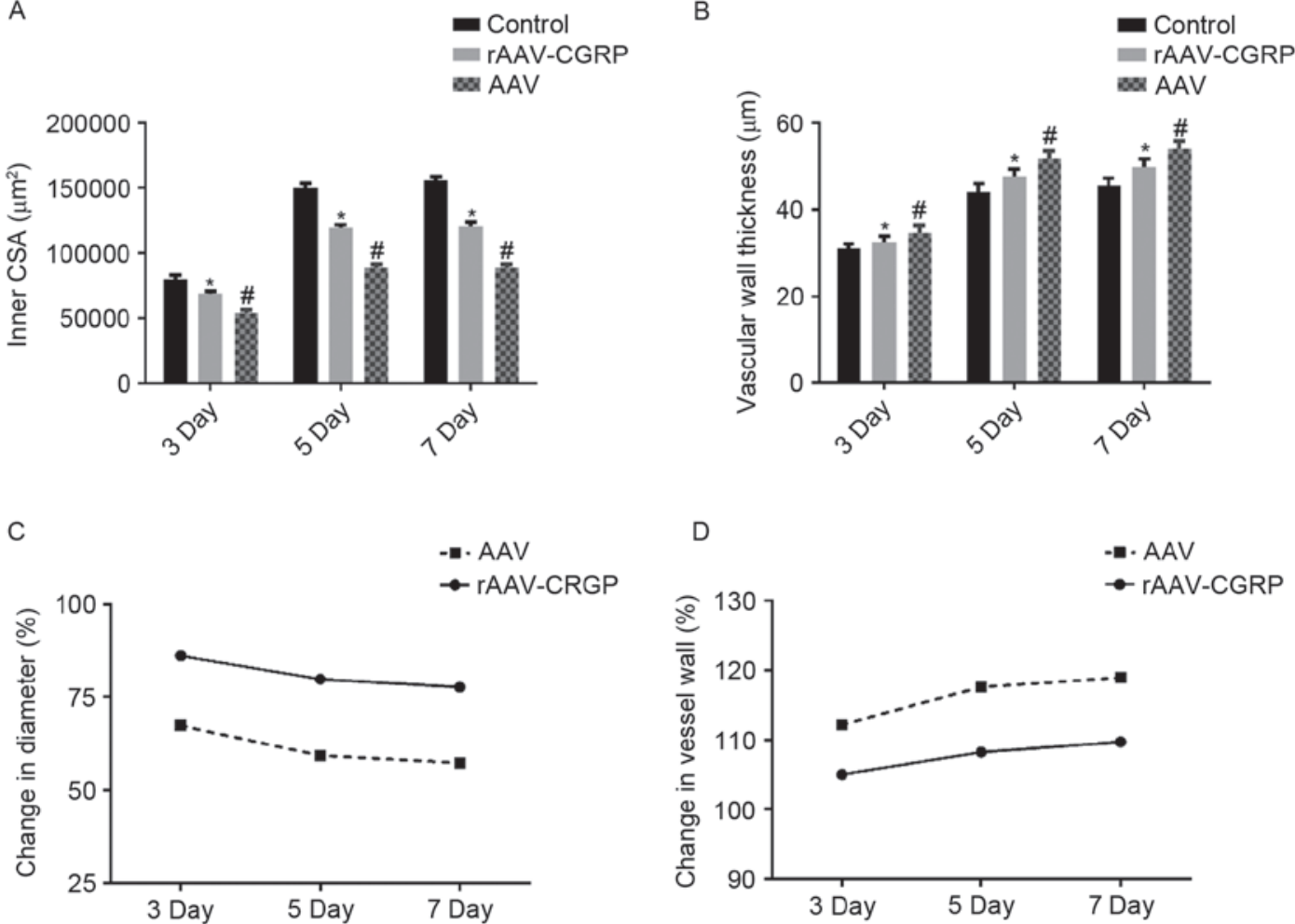

D

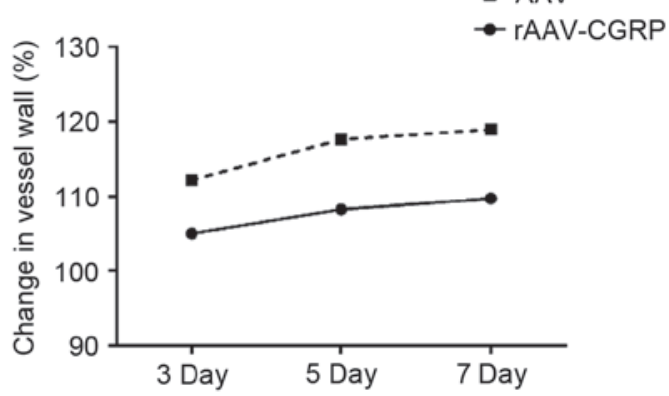

Figure 3. Inner CSA and vessel wall thickness of the umbilical artery in all groups, and the changing trends of the rAAV-GPCR and AAV groups, compared with that of the control group. (A) Inner CSA of the umbilical artery in the allantoic cavity in all groups ( $\left.\mu \mathrm{m}^{2}\right)$. (B) Vessel wall thickness of the umbilical arteries in the allantoic cavity in all groups $(\mu \mathrm{m})$. (C) The changing trend in the mean inner CSA of the umbilical artery in the rAAV-GPCR and AAV groups compared with those in the control group. (D) Changing trend in vessel wall thickness in the rAAV-GPCR and AAV groups compared with that of the control group. ${ }^{*} \mathrm{P}<0.05$ vs. the control group; ${ }^{~} \mathrm{P}<0.05$ vs. the rAAV-CGRP and control groups. CSA, cross-sectional area; rAAV, recombinant adeno-associated virus; CGRP, calcitonin gene-related peptide; AAV, adeno-associated virus.

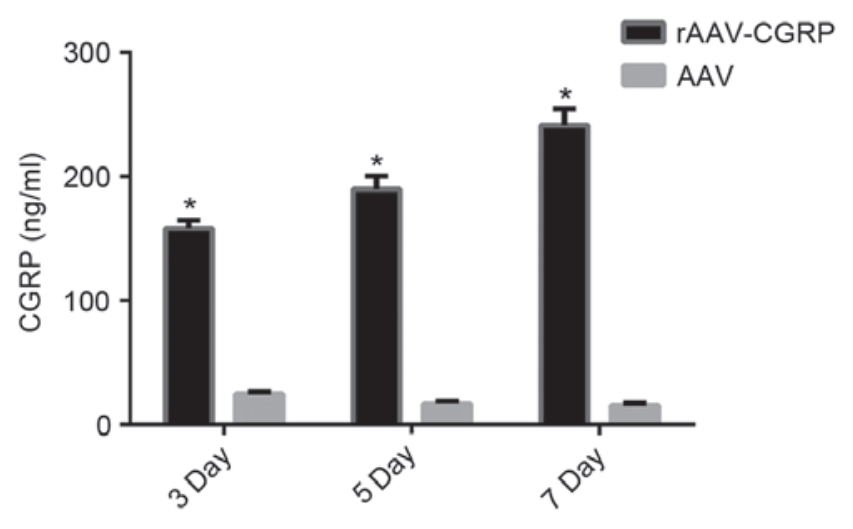

Figure 4. CGRP concentrations in the allantoic cavity fluid in the rAAV-CGRP and AAV groups. CGRP concentrations in the allantoic cavity fluid after 3,5 and 7 days of model establishment in the rAAV-CGRP and empty vector AAV groups. CGRP concentrations in the rAAV-CGRP group were significantly higher than those in the empty vector AAV group. ${ }^{*} \mathrm{P}<0.05$ vs. the AAV group. CGRP, calcitonin gene-related peptide; rAAV, recombinant adeno-associated virus; $\mathrm{AAV}$, adeno-associated virus.

the improvement of CVS and recovery of neurological function within a short period (12-14). However, the intravenous application of CGRP easily induced complications, such as hypotension and increased heart rate (12-14). Furthermore, due to its protein features, CGRP has a short plasma half-life of only 7-10 $\mathrm{min}$. These phenomena have limited the clinical application of CGRP (15).
With the development of genetic engineering technology, studies on CGRP gene therapy for CVS after SAH have received more attention and have become a hot topic in clinical studies. Toyoda et al (25) injected the CGRP gene mediated by an adenovirus into the cisterna magna of rabbits in a SAH model. After five days, the CGRP concentration in the CSF of the CGRP transgene treatment group increased 400-fold, the basilar artery diameter increased by $25 \%$, and the degree of spasm significantly improved compared with the values in the control group. Satoh et al (26) applied the same method in a canine CVS model. Compared with the control group, the CGRP concentration in the CSF of the CGRP transgene treatment group increased 115-fold and the basilar artery diameter increased by $25 \%$. These results further confirmed that adenovirus-mediated CGRP gene therapy improves CVS after SAH. Tian et al (7) injected Tat-gelatin siloxane nanoparticles encapsulating the expression plasmid vector $\mathrm{pLXSN-CGRP}$ into the cisterna magna of a CVS model in rats. Compared with the control group, the CGRP expression levels in the CSF of the treatment group increased, the perimeter of the basilar artery lumen increased by $23 \%$, the vessel wall thickness decreased by $76 \%$ and the neurological function significantly improved. These findings support the feasibility of CGRP gene therapy in CVS after SAH.

In the present study, the CGRP gene was cloned into AAV using genetic engineering technology to construct a eukaryotic expressing vector, rAAV-CGRP, containing the CGRP gene. Through the allantoic cavity injection route, the feasibility of 
improving umbilical artery vasospasm in the chick embryo allantoic cavity induced by allantoic cavity hemorrhage using rAAV-CGRP was demonstrated. The embryonic chick allantois is a sac formed by a bulge from the ventral side of the embryo during chick embryo incubation; this sac is filled with allantoic fluid. Internally, it is wrapped with umbilical arteries and veins. The allantoic umbilical artery travels through the allantoic cavity and is divided into two branches. After entering the CAM, the allantoic umbilical artery gradually branches to form a dense CAM vascular network, which gradually converges into the CAM veins and finally returns to the body through the CAM veins $(27,28)$. The natural fluid environment of the umbilical arteries in the allantoic cavity is similar to the subarachnoid CSF environment of cerebral blood vessels in the skull base. Allantoic umbilical artery spasm induced by allantoic cavity hemorrhage using CAM vein puncture is able to simulate the pathological changes of CVS after SAH. These phenomena have been confirmed in our previous studies $(16,27)$. As a commonly used gene transfer vector, AAV has the advantages of excellent safety, a broad host range, a specific integration function, and long-term stable expression of exogenous genes; therefore, AAV is widely used in gene therapy experimentation of mammalian CVS models $(25,26)$ and can be used for the stable expression of gene vectors in chick embryos $(29,30)$.

The present study injected constructed rAAV-CGRP into the chick embryo allantoic cavity after $24 \mathrm{~h}$ of establishment of the chick embryo umbilical artery vasospasm model. The CGRP concentrations in the allantoic fluid after 3, 5 and 7 days of model establishment were detected using ELISA reagent kits. The results showed that the CGRP concentration in allantoic fluid in the rAAV-CGRP group significantly increased and exhibited a gradual increasing trend compared with that of the empty vector AAV group. The CGRP concentration in the allantoic fluid in the empty vector AAV group exhibited a decreasing trend. These results indicated that $\mathrm{rAAV}-\mathrm{CGRP}$ was stably expressed in chick embryos; the CGRP concentration in the allantoic fluid gradually increased, whereas endogenous CGRP in the empty vector AAV group gradually depleted. These results were consistent with previous studies in mammals $(7,25,26)$. Pathological sections of umbilical arteries also revealed that the chick embryo allantoic umbilical artery developed severe vasospasm in the model group injected with empty vector AAV. On days 3, 5 and 7 after model establishment, the inner CSA of the umbilical arteries were only 67.33, 59.19 and $57.22 \%$, respectively, of those in the age-matched control group, and the vessel wall thickness of the umbilical arteries increased by $12.17,17.59$ and $18.95 \%$, respectively, of those in the age-matched control group. In the rAAV-CGRP injection group, on days 3, 5, and 7 after model establishment, the degrees of vasospasm significantly improved, and the inner CSA of the umbilical arteries were only 86.11 , 79.75 and $77.60 \%$, respectively, of those in the age-matched control group; similarly, the vessel wall thickness values of the umbilical arteries increased to 5.00, 8.21 and 9.73\%, respectively, of those in the age-matched control group. The above results suggested that rAAV-CGRP significantly increased CGRP expression levels in the chick embryo allantoic cavity and improved umbilical artery vasospasm induced by allantoic cavity hemorrhage. However, rAAV-CGRP was not able to completely reverse the vasospasm resulting from allantoic cavity hemorrhage caused by puncture. In addition, the statistical results of the successful rate of umbilical artery vasospasm models induced by chick embryo allantoic cavity hemorrhage demonstrated that, after $72 \mathrm{~h}$ of model establishment, the control group contained one chick embryo that did not survive in the 7-day subgroup. Following model establishment, the mortality rates in the 3-, 5- and 7-day subgroups of the rAAV-CGRP group were 25, 25 and $37.5 \%$, respectively, which were lower than the values of $50,37.5$ and $62.5 \%$, respectively, in the age-matched subgroups of the empty vector AAV group. The differences between these two groups were not statistically significant. However, the mortality rates in the 3- and 7-day subgroups in the chick embryo model of the empty vector AAV group were significantly higher than those in the control group. These results suggest that injection of rAAV-CGRP through the allantoic cavity may improve the oxygen supply and blood supply during chick embryo development and increase the success rate of the umbilical artery vasospasm model induced by chick embryo allantoic cavity hemorrhage, possibly by relieving umbilical artery vasospasm.

The present study used an umbilical artery vasospasm model induced by chick embryo allantoic cavity hemorrhage to validate the effectiveness of rAAV-CGRP treatment. This model had the advantages of simple manipulation, ease of feeding, low cost, short cycles, and fewer ethical restrictions compared with previous mammalian vasospasm models. Notably, although AAV is a commonly used vector for gene therapy of CVS in mammalian models and has high transfection efficiency, it induces cytotoxicity and immunogenicity; in particular, it may induce a systemic toxic reaction or immune and inflammatory reactions $(25,26)$ that interfere with experimental results. The deficiency of innate immunity in chick embryos may compensate for this shortcoming $(31,32)$. The present study also had certain limitations. The umbilical artery vasospasm model induced by chick embryo allantoic cavity hemorrhage would not be able to completely simulate vasospasm after SAH in human experimentation. Compared with mammalian models, the model used in this study did not evaluate the neurologic impairment by behavior after rAAV-CGRP treatment, and the treatment results could only be evaluated by pathological change.

In conclusion, the present study demonstrated that rAAV-CGRP is able to increase CGRP expression in the allantoic cavity in a vasospasm model induced by allantoic cavity hemorrhage in the chick embryo, improve vasospasm induced by allantoic cavity hemorrhage, and increase the success rate of the vasospasm model induced by allantoic cavity hemorrhage in the chick embryo. These results further validate the feasibility of gene therapy for CVS after SAH. In addition, the utilization of this simple vasospasm model induced by allantoic cavity hemorrhage in the chick embryo may reduce the use of mammalian SAH models and reduce experimental costs. This model also has the advantage of natural immunity deficiency to prevent the inflammatory response of viral vectors. Although there are certain limitations, this model may be used as a simple and low-cost vasospasm model for preliminary studies to identify other target genes in the pursuit of effective gene therapy. 


\section{Acknowledgements}

This study was supported by the National Natural Science Foundation of China (grant no. 81200888).

\section{References}

1. Becker KJ: Epidemiology and clinical presentation of aneurysmal subarachnoid hemorrhage. Neurosurg Clin N Am 9: 435-444, 1998.

2. Przybycien-Szymanska MM and Ashley WW Jr: Biomarker discovery in cerebral vasospasm after aneurysmal subarachnoid hemorrhage. J Stroke Cerebrovasc Dis 24: 1453-1464, 2015

3. Budohoski KP, Guilfoyle M, Helmy A, Huuskonen T, Czosnyka M, Kirollos R, Menon DK, Pickard JD and Kirkpatrick PJ: The pathophysiology and treatment of delayed cerebral ischaemia following subarachnoid haemorrhage. J Neurol Neurosurg Psychiatry 85: 1343-1353, 2014.

4. Gathier CS, vandenBergh WMand Slooter AJ; HIMALAIA-Study Group: HIMALAIA (Hypertension Induction in the Management of AneurysmaL subArachnoid haemorrhage with secondary IschaemiA): A randomized single-blind controlled trial of induced hypertension vs. no induced hypertension in the treatmen of delayed cerebral ischemia after subarachnoid hemorrhage. Int J Stroke 9: 375-380, 2014

5. Francoeur CL and Mayer SA: Management of delayed cerebral ischemia after subarachnoid hemorrhage. Crit Care 20: 277, 2016.

6. Sun BL, Shen FP, Wu QJ, Chi SM, Yang MF, Yuan H, Xie FM, Zhang YB, Chen J and Zhang F: Intranasal delivery of calcitonin gene-related peptide reduces cerebral vasospasm in rats. Front Biosci (Elite Ed) 2: 1502-1513, 2010.

7. Tian XH, Wang ZG, Meng H, Wang YH, Feng W, Wei F, Huang ZC, Lin XN and Ren L: Tat peptide-decorated gelatin-siloxane nanoparticles for delivery of CGRP transgene in treatment of cerebral vasospasm. Int J Nanomedicine 8: 865-876, 2013.

8. Kolias AG, Sen J and Belli A: Pathogenesis of cerebral vasospasm following aneurysmal subarachnoid hemorrhage: Putative mechanisms and novel approaches. J Neurosci Res 87: 1-11, 2009.

9. Hu N, Wu Y, Chen BZ, Han JF and Zhou MT: Protective effect of stellate ganglion block on delayed cerebral vasospasm in an experimental rat model of subarachnoid hemorrhage. Brain Res 1585: 63-71, 2014

10. Nozaki K, Uemura Y, Okamoto S, Kikuchi H and Mizuno N Relaxant effect of calcitonin gene-related peptide on cerebral arterial spasm induced by experimental subarachnoid hemorrhage in dogs. J Neurosurg 71: 558-564, 1989.

11. Toshima M, Kassell NF, Tanaka Y and Dougherty DA: Effect of intracisternal and intravenous calcitonin gene-related peptide on experimental cerebral vasospasm in rabbits. Acta Neurochir (Wien) 119: 134-138, 1992.

12. Juul R, Aakhus S, Bjornstad K, Gisvold SE, Brubakk AO and Edvinsson L: Calcitonin gene-related peptide (human alpha-CGRP) counteracts vasoconstriction in human subarachnoid haemorrhage. Neurosci Lett 170: 67-70, 1994.

13. Juul R, Edvinsson L, Gisvold SE, Ekman R, Brubakk AO and Fredriksen TA: Calcitonin gene-related peptide-LI in subarachnoid haemorrhage in man. Signs of activation of the trigemino-cerebrovascular system? Br J Neurosurg 4: 171-179, 1990.

14. Effect of calcitonin-gene-related peptide in patients with delayed postoperative cerebral ischaemia after aneurysmal subarachnoid haemorrhage. European CGRP in Subarachnoid Haemorrhage Study Group. Lancet 339: 831-834, 1992

15. Kokkoris S, Andrews P and Webb DJ: Role of calcitonin gene-related peptide in cerebral vasospasm, and as a therapeutic approach to subarachnoid hemorrhage. Front Endocrinol (Lausanne) 3: 135, 2012.

16. Yuan Y, Yang S, Li C, Xu K, Luo Q and Yu J: The chicken embryo umbilical artery is a promising in vivo model system for the study of vasospasm. Int J Clin Exp Med 9: 1139-1149, 2016

17. Pallás V, Más P and Sánchez-Navarro JA: Detection of plant RNA viruses by nonisotopic dot-blot hybridization. Methods Mol Biol 81: 461-468, 1998.
18. Edvinsson L, Ekman R, Jansen I, McCulloch J, Mortensen A and Uddman R: Reduced levels of calcitonin gene-related peptide-like immunoreactivity in human brain vessels after subarachnoid haemorrhage. Neurosci Lett 121: 151-154, 1991.

19. Schebesch KM, Herbst A, Bele S, Schödel P, Brawanski A, Stoerr EM, Lohmeier A, Kagerbauer SM, Martin J and Proescholdt M: Calcitonin-gene related peptide and cerebral vasospasm. J Clin Neurosci 20: 584-586, 2013.

20. Juul R, Hara H, Gisvold SE, Brubakk AO, Fredriksen TA, Waldemar G, Schmidt JF, Ekman R and Edvinsson L: Alterations in perivascular dilatory neuropeptides (CGRP, SP, VIP) in the external jugular vein and in the cerebrospinal fluid following subarachnoid haemorrhage in man. Acta Neurochir (Wien) 132: 32-41, 1995.

21. Edvinsson L, Delgado-Zygmunt T, Ekman R, Jansen I, Svendgaard NA and Uddman R: Involvement of perivascular sensory fibers in the pathophysiology of cerebral vasospasm following subarachnoid hemorrhage. J Cereb Blood Flow Metab 10: 602-607, 1990.

22. Imaizumi S, Shimizu H, Ahmad I, Kaminuma T, Tajima M and Yoshimoto T: Effect of calcitonin gene-related peptide on delayed cerebral vasospasm after experimental subarachnoid hemorrhage in rabbits. Surg Neurol 46: 263-271, 1996.

23. Ahmad I, Imaizumi S, Shimizu H, Kaminuma T, Ochiai N, Tajima $M$ and Yoshimoto T: Development of calcitonin gene-related peptide slow-release tablet implanted in CSF space for prevention of cerebral vasospasm after experimental subarachnoid haemorrhage. Acta Neurochir (Wien) 138: 1230-1240, 1996

24. Inoue T, Shimizu H, Kaminuma T, Tajima M, Watabe $\mathrm{K}$ and Yoshimoto T: Prevention of cerebral vasospasm by calcitonin gene-related peptide slow-release tablet after subarachnoid hemorrhage in monkeys. Neurosurgery 39: 984-990, 1996.

25. Toyoda K, Faraci FM, Watanabe Y, Ueda T, Andresen JJ, Chu Y, Otake S and Heistad DD: Gene transfer of calcitonin gene-related peptide prevents vasoconstriction after subarachnoid hemorrhage. Circ Res 87: 818-824, 2000.

26. Satoh M, Perkins E, Kimura H, Tang J, Chun Y, Heistad DD and Zhang JH: Posttreatment with adenovirus-mediated gene transfer of calcitonin gene-related peptide to reverse cerebral vasospasm in dogs. J Neurosurg 97: 136-142, 2002.

27. Yuan YJ, Xu K, Wu W, Luo Q and Yu JL: Application of the chick embryo chorioallantoic membrane in neurosurgery disease. Int J Med Sci 11: 1275-1281, 2014

28. Tufan AC and Satiroglu-Tufan NL: The chick embryo chorioallantoic membrane as a model system for the study of tumor angiogenesis, invasion and development of anti-angiogenic agents. Curr Cancer Drug Targets 5: 249-266, 2005.

29. Tutykhina IL, Bezborodova OA, Shmarov MM, Logunov DY, Neugodova GL, Nemtsova ER, Naroditsky BS, Yakubovskaya RI and Gintsburg AL: Production of recombinant human lactoferrin in the allantoic fluid of embryonated chicken eggs and its characteristics. Protein Expr Purif 65: 100-107, 2009.

30. Wang Y, Sun H, Shen P, Zhang X and Xia X: Effective inhibition of infectious bursal disease virus replication by recombinant avian adeno-associated virus-delivered microRNAs. J Gen Virol 90: 1417-1422, 2009.

31. Ribatti D: Chicken chorioallantoic membrane angiogenesis model. Methods Mol Biol 843: 47-57, 2012.

32. Palmer TD, Lewis J and Zijlstra A: Quantitative analysis of cancer metastasis using an avian embryo model. J Vis Exp: 2815, 2011.

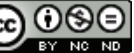

This work is licensed under a Creative Commons Attribution-NonCommercial-NoDerivatives 4.0 International (CC BY-NC-ND 4.0) License. 